\title{
An Investigation into Mobile Application Development Processes: Challenges and Best Practices
}

\author{
Harleen K. Flora \\ The IIS University, Jaipur, India \\ harleenflora@gmail.com \\ Xiaofeng Wang \\ Free University of Bozen/Bolzano, Italy \\ xiaofeng.wang@unibz.it \\ Swati V. Chande \\ International School of Informatics and Management, Jaipur, India \\ swati.v.chande@iisuniv.ac.in
}

\begin{abstract}
The mobile device market has witnessed swift industrial growth over the last decade. The quick expansion of this new computing platform has almost outpaced the software engineering processes customized to mobile application development. However, there is still lack of novel research initiatives around the mobile application development process. There remains a deficiency in development standards and best practices which expose the mobile device to potential attacks. This deficiency needs to be addressed promptly and requires further work.

The objective of this research is to better understand the current methodologies adapted and to investigate challenges faced during the mobile application development processes that are different from traditional enterprise application. For this purpose, an online survey was conducted from the mobile research and development community. The survey questions covered the entire mobile application development lifecycle starting with requirements, and ending with bringing to life a complete mobile application.

The study contributes towards a greater understanding of mobile application development process, examines real challenges confronted, and investigates the best practices that can be successfully implemented to enhance, evaluate, and improve the performance of the mobile application development process. These findings can also be considered as a possible research topic that indicates the breadth of research requirements and prospects in mobile computing.
\end{abstract}

Index Terms - Mobile devices, mobile apps, mobile app development, mobile computing, mobile issues and challenges, mobile development best practices

\section{INTRODUCTION}

As mobile platforms continue to advance in performance, users are expecting their mobile devices to provide functionality similar to their desktop computer applications. However, the development of mobile applications (mobile apps) is still considered to be complex, and various methodologies adopted towards the development of such technologies is inadequate.

Traditional software engineering approaches and methods used in the development of desktop applications may not be directly applicable to a mobile environment $[1,2]$. Therefore, it is critical to develop and adopt appropriate methodologies for the development of mobile applications as there are a number of key issues and challenges that are different from traditional enterprise applications.

However, the development of mobile software is still unwieldy and a methodology geared towards supporting the development of such mobile applications is still inadequate. There is still lack of research initiatives and insufficient understanding of real issues and challenges faced during the development of mobile apps. This exposes the mobile device to prospective attacks which need to be addressed promptly and require further work that motivates this study.

The current paper addresses these key questions systematically. The objective of our study was to gain a better understanding of the mobile application development process currently adopted by mobile developers worldwide and the various issues and challenges associated with it. For this purpose, an online survey was conducted from the mobile development and research community. The current study aims at 
answering the following three questions: 1) what are the current methodologies adapted during the mobile application development process? 2) What are the issues and challenges that are faced during the development of mobile applications? 3) What are the best practices various organizations have adopted and suggested to mitigate the challenges that can be faced during early development process?

The paper is organized as follows. Section I presents the introduction. Section II discusses the related work. Section III explains the research approach used for this study. Section IV describes the findings of this study by classifying the issues and challenges of the mobile application development process followed by mobile developers. Finally, Section V is discussion and Section VI concludes the paper and outlines future work.

\section{LITERATURE REVIEW}

Mobile Application Development (MAD) is the process by which applications are developed for small low-power handheld devices. Though the mobile application development process is similar to software engineering, it also presents some additional requirements for which the traditional software development process has to be customized $[1,2]$. The key characteristics and features that differentiate a mobile version from a conventional desktop application can be grouped into three categories: i.e. Hardware, Software and Communication [3, 4].

There are number of major things found when looking at the end-to-end process of developing a mobile application, from business discovery and development to support and marketing. To date, few studies have identified and published the fundamental challenges in mobile computing. Leigh Williamson [1] listed the unique challenges for mobile application development, such as form factors and user input technology, usability and user interaction design, and choice of implementation technology for native, web and hybrid mobile app implementation.

Wasserman et. al. [2] identified issues related to mobile application development based upon development processes, tools, user interface design, application portability, quality, and security.

Dehlinger et al. [3] identified four main challenges for mobile application software engineering. They observed these challenges particularly when creating universal user interfaces, when enabling software reuse across mobile platforms, when designing context-aware mobile apps, and while balancing agility and uncertainty in requirements

In addition, Dye et al. [5] discussed various security challenges as a result of the abundance of mobile software applications in recent years and conversed about the potential risks that these devices are exposed to due to lack of development standards and best practices.

These issues are imperative and should be considered during the early development process in order to mitigate the impact of poor choices. However, very few reports have investigated and highlighted the best practices for mobile application development projects. The largest and most complicated software and system development projects have moved away from a process-intensive approach toward a more agile approach. The Scrum approach, for example is an iterative and incremental frame-work commonly used in combination with other Agile techniques like test driven development, continuous integration and testing are utilized in today's mobile application development $[6,7,8]$.

\section{RESEARCH APPROACH}

A research survey was conducted for this study to gain valuable inputs regarding various emergent practices during the mobile application development process. The results of the survey were utilized as a starting point to identify specific requirements in the area of mobile software engineering for new and revolutionary business practices. An extensive online questionnaire was designed based on the available literature related to mobile app development and also by evaluating several online surveys conducted in the past by various commercial organizations. Main contributors to the survey were mobile companies, mobile development team members, mobile experts, researchers, and relevant stakeholders.

The participants were invited to contribute in the survey on a voluntary basis via various mobile app development forums. The design of the questionnaire was evaluated and validated by several experts in the area before it was issued to the participants. The data was analysed quantitatively and qualitatively by studying each response individually and then by analysing all responses collectively. The validity of the study and findings was assessed by relevant experts from mobile development community. More than 130 responses were received during the data collection period. In this paper we report the part of the results that are pertinent to the issues, challenges, and best practices of mobile software development processes.

Figures 1 to 5 below represent the general information regarding the demographic characteristics and experience of the participants in the area of mobile application development. It can be noted from the figures below that $69 \%$ of respondents comes from the mobile industry with actual mobile app development experience. These participants cover different continents, with the biggest group (38\%) coming from North America and 25\% of organization size that comprises of 5-25 members.

Maximum response was received from development team members (47\%) as compared to consultant/trainers $(33 \%)$ and top level management $(20 \%)$ with relatively more experience in developing mobile softwares.

The developer participants mainly expertise in mobile development categories related to Education (14\%), Utilities (14\%) and communication (13\%). 


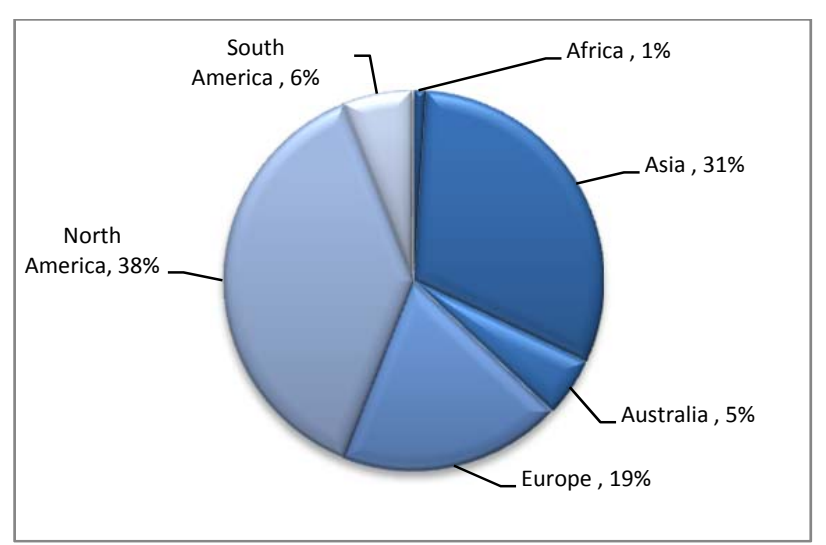

Fig. 1: Organization Location.

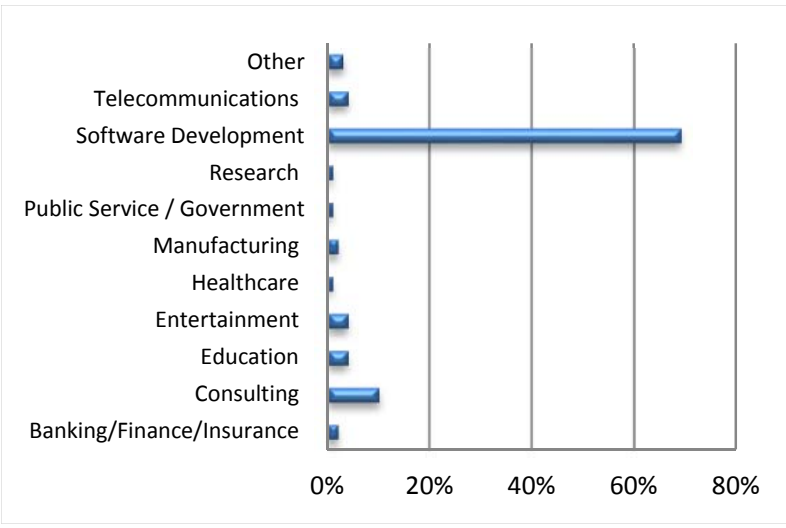

Fig. 2: Organization Type

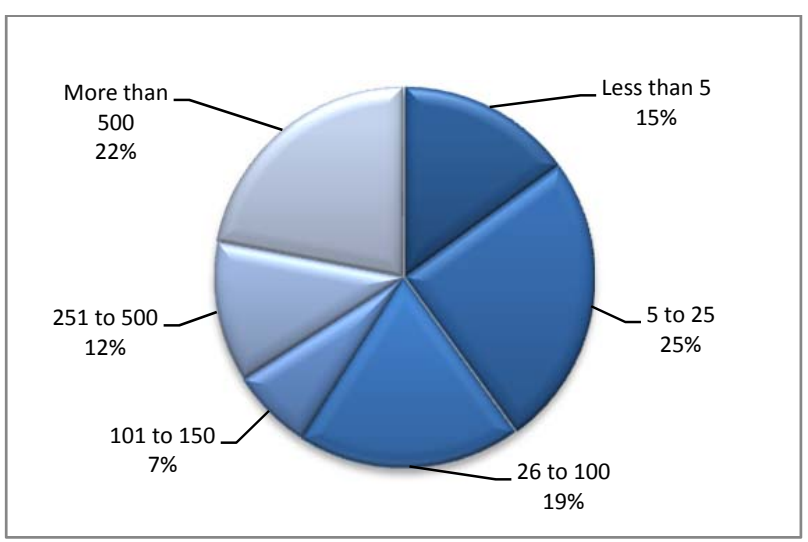

Fig. 3: Organization Size.

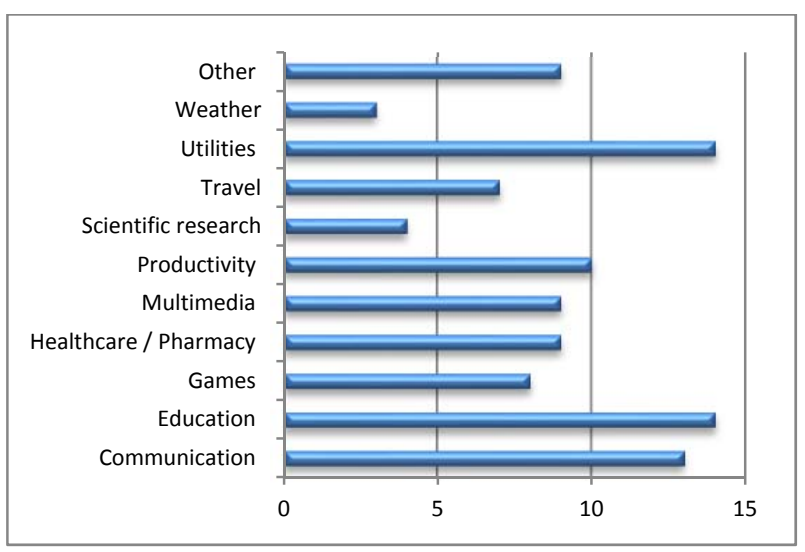

Fig. 4: Categories of Mobile Applications Developed.

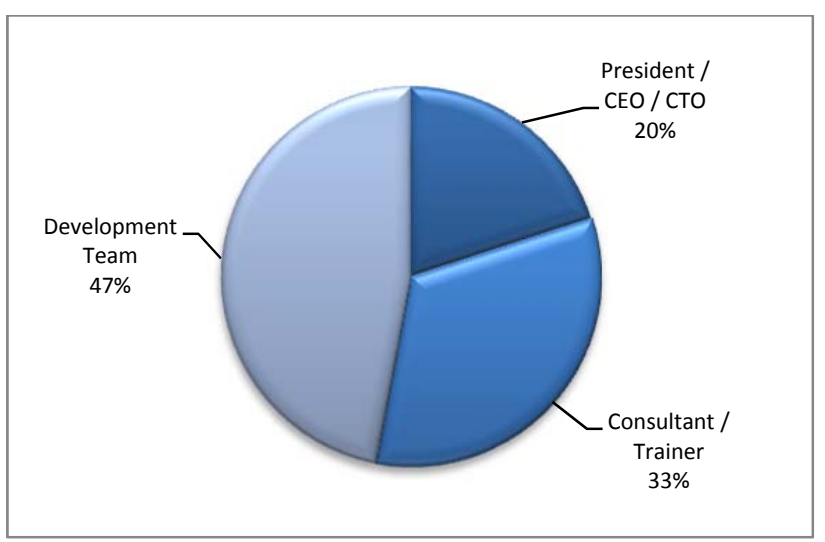

Fig. 5: Respondent Role in Organization.

\section{FINDINGS}

\section{A. Mobile Application Development - Process}

Figure 6 below state the reasons why the participants started mobile application development. $22 \%$ of participants reported that their motivation for building mobile apps is for the extension of the company.

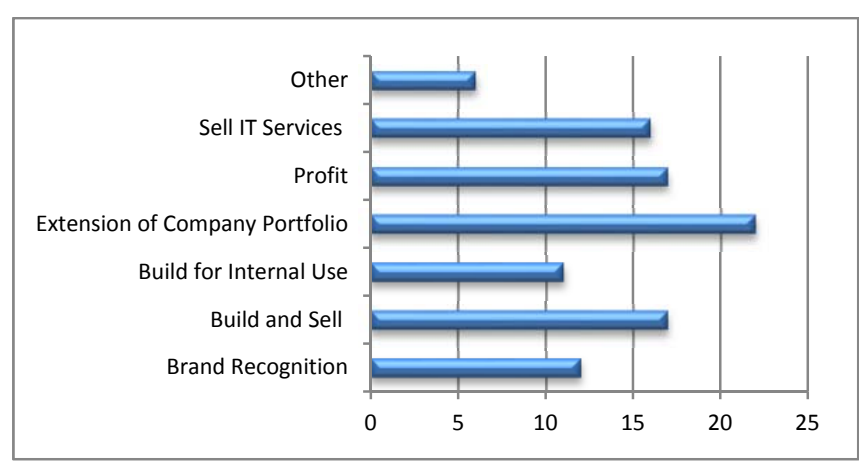

Fig. 6: Motivation for building mobile apps.

As shown in Figure 7, 36\% of survey participants indicated that mainly large businesses commission their apps.

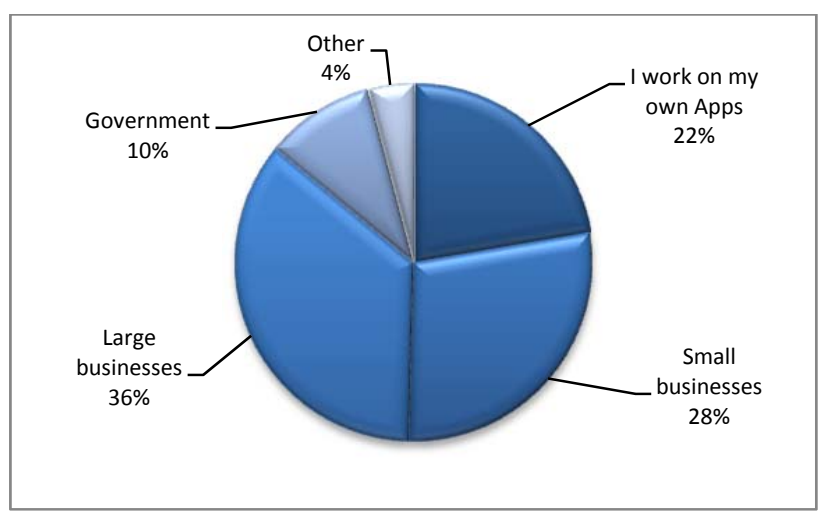

Fig. 7: Who commissions the app?

It can be seen from the below figures $8-13$ that $41 \%$ of participants belonged to medium sized development teams (5-15 members). $57 \%$ of mobile developers claim 
that it generally takes 6-18 weeks to develop a mobile app. Regarding application testing, 97\% of participants claimed that their organizations test mobile apps before releasing, $46 \%$ said that their mobile app production release frequency is usually on monthly basis, and $74 \%$ of participants test mobile apps for 1-2 weeks before production release.

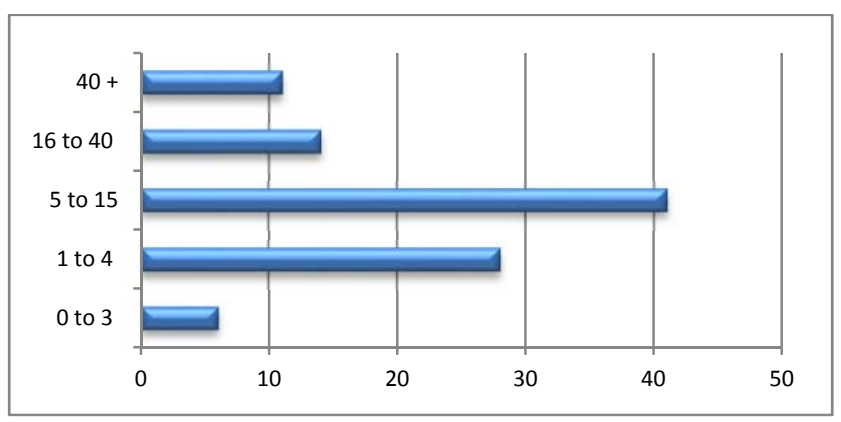

Fig. 8: Team Size.

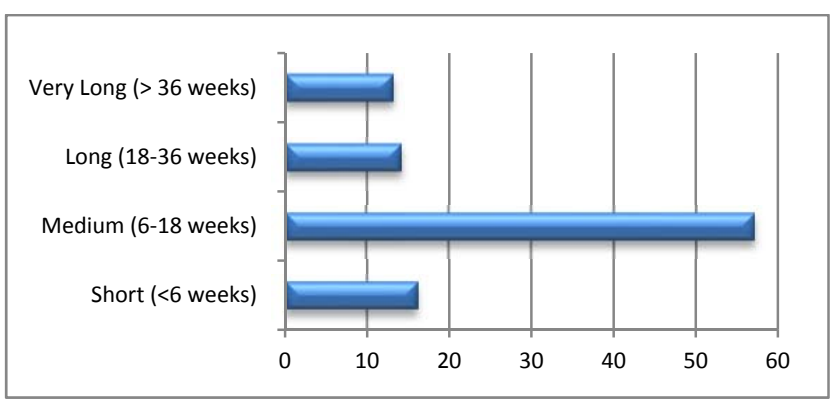

Fig. 9: Development process duration.

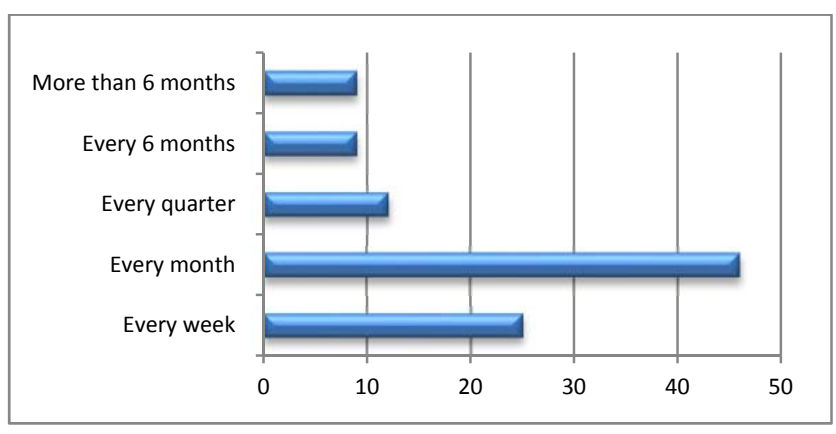

Fig. 10: Production release frequency.

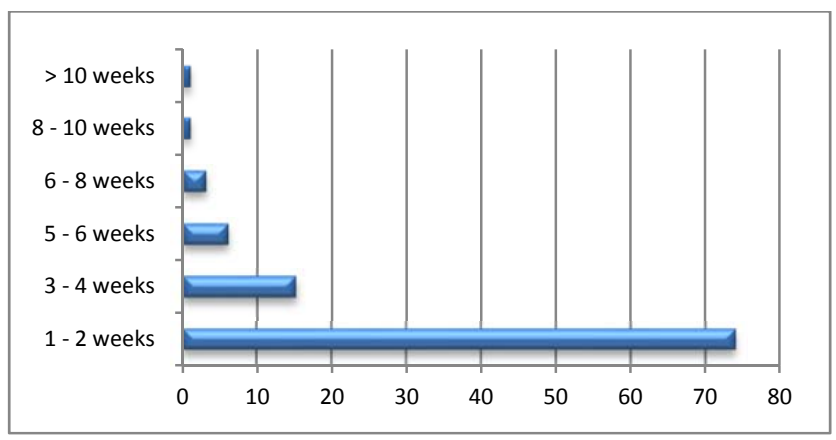

Fig. 11: Test Duration.

Above $50 \%$ of participants believed that the development process of mobile app differs from other types of software. It can be seen from below figures that the developers are more confident in developing mobile

Copyright (C) 2013 MECS apps using Java (22\%), Objective C /HTML5 (15\%) and JavaScript (14\%). Android (35\%), iOS Apple (33\%) and Windows Mobile (12\%) show strong interest from developers out of a variety of available platforms.

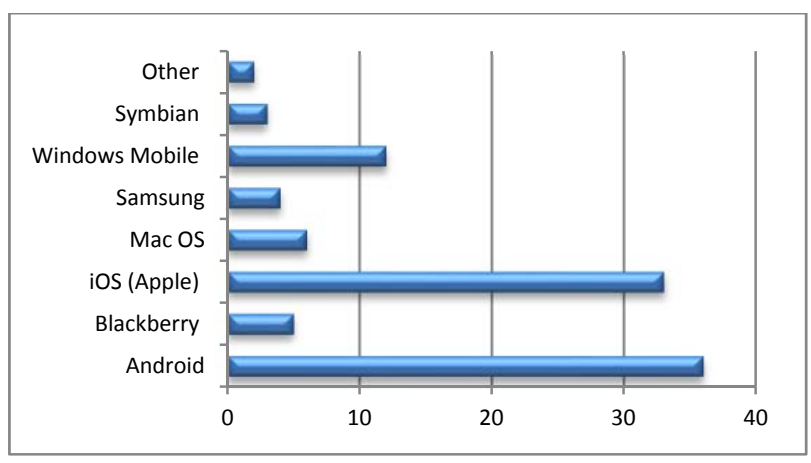

Fig. 12: Platform used for development.

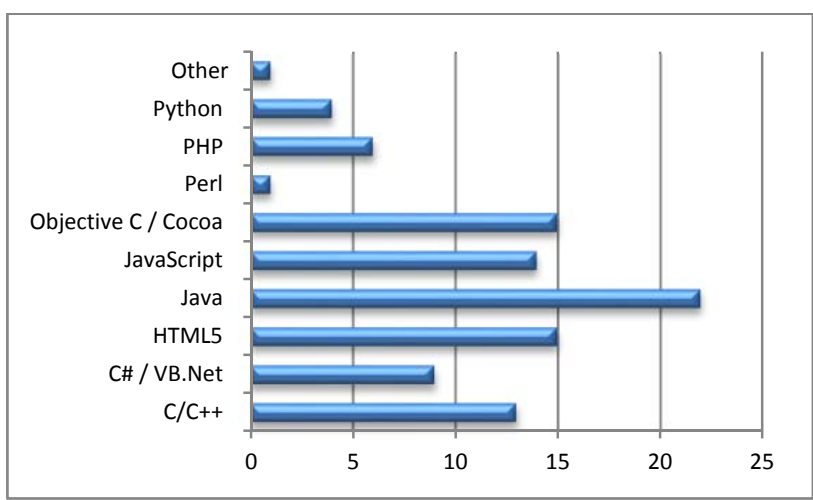

Fig. 13: Languages used for development.

This section discusses a flexible approach towards mobile application development starting from concept to release. The process will not only help to resolve user's requirement and ideas for new releases, but will also help to streamline the mobile software lifecycle.

After reviewing and analyzing the survey responses, the mobile software engineering process can subdivided into the following four major phases. These phases have been further endorsed by mobile experts as a proven way to develop systematic and successful mobile applications starting from concept to release.

\section{Phase 1: Envision}

Analysis: Participants emphasized that the creation of an exceptional mobile application starts by identifying the mobile initiatives, problem, purpose, goal, and audience for which the application will be developed. The Envision phase should start with proper understanding of user's vision and business requirements followed by deciding the application features and addressing the exact services the application will offer to the business.

Planning: The absolute goal of planning stage is to ensure that product creation objectives, mobile technology, and innovative design requirements are captured properly and communicated among team members. For this purpose, it is suggested that it should 
commence with the roadmap of mobile product layout that includes estimate planning, strategy planning, and user experience design, followed by identification of exact solution.

Estimate planning is prepared for higher level assessment of budget and schedule, selection of platforms, devices and browsers, sprint and release planning, and developing an initial vision. Strategy planning defines target, goals, monetization by considering project feasibility. User Experience (UX) design is accomplished by proposing wireframes, storyboards and sketches, using strategies to make code optimized, by optimizing the memory issues, defining precise UI description, defining work breakdown structure of functionalities into smaller user stories and tasks. And identification of exact solution is based on criteria, application target user and user domain, mobile platforms and types of devices to be supported, databases and multi-tenancy and Revenue Model.

The Project Plan should be further broken into modules, work breakdown structures, milestones, required development resources, and detail mobile product specifications.

\section{Phase 2: Solution}

Design: The design stage creates a visual design view, mockup screens, and look and feel of the app for clients' approval to save valuable time before starting the actual coding process. It is suggested that the process should contain design specifications including detailed model level design specification; creation of user interface step models and security models; architecture including the creation of initial prototypes and mockups with graphics integration. And, confirming the UI design upon user's approval by constructing wireframes that demonstrate major scenarios and how the user interacts with the application followed by a test plan that includes writing story cards, test plan, and test codes.

Development: Upon clients design approval, the actual development process of an app should begin with iterations following the project plan. As stated by participants, they practice Sprint based development that contains writing codes by breaking the projects in modules, and by developing backend and database designing (if required). Testing iteratively is highly recommended as the code should be tested on a reference device by executing unit test, bug fixing, and intermediate release for clients testing. Once developed, the entire project should be available for clients review and testing.

\section{Phase 3: Quality Assurance}

Testing: After design and development stage, the application should be tested with the project plan, requirements, specifications, wireframes and designs created at the earlier stages. As per expert mobile tester participants, the testing stage should consist of defining test cases, automated testing, and finally testing on emulator. The test cases should be defined as per module standalone test specification, integrated and system test specifications. Automated testing should be used to the highest degree possible. Regression testing in addition to unit testing should be integrated into a continuous integration environment so that application remains in a consistently tested state. And finally testing on emulator and over wide variety of devices should be done for usability, functionality, compatibility, performance, interoperability, security, localization, and connectively.

Change: After user acceptance testing (UAT), changes as suggested by users and final bug fixes should be integrated followed by final user testing and client's approval.

\section{Phase 4: Product Release}

Deployment: After final testing and approval from clients, participants from the mobile app development team started deployment preparation by installing the created mobile app on the server and submitting it to the app store. This is followed by product marketing, launching a beta test, optimizing retention, new users on boarding, flow of subscriptions, and launching the $1^{\text {st }}$ version of a newly created mobile app.

Support and Maintenance: Most of the survey participants highly emphasized the necessity to capture the defects based upon user-market feedbacks, through memory optimization, automated crash reports, and change requests from users. They highly recommended that product maintenance (support) and product improvement (upgrade) should be done in frequent iterative releases with bug fixes via app store or enterprise deployment, This support should be improving the app with platform updates, new features, and functionalities.

\section{B. Mobile Application Development - Issues and Challenges}

The increasing complexity of handheld devices, the escalating market for apps, and the growing competence of wireless networks all work together in making mobile app development an industry with massive potential. Mobile applications development has become the support system of the mobile communication system, and with the continuing increase in number of hardware and software, the number of challenges faced also increases. Careful planning and execution of a well-defined mobile application development strategy can lead to significant gains in key business drivers. However, there are still a number of challenges which are currently addressed by researchers, academicians, and practitioners in the field.

In this section, we present the unique requirements, most prominent issues, and challenges faced by mobile developers emerging from our study results related to hardware and software issues while building a mobile application.

\section{Challenges related to Hardware}


In this subsection, we present the most prominent challenges faced by mobile app developers related to Hardware issues emerging from our study results:

Cross platform compatibility: With a myriad of mobile platforms available currently, mobile companies have to design and develop their apps to run on multiple devices and offer cross platform compatibility. Due to the highly fragmented nature of hardware, software, tool and technology needed to build apps on various platforms, $45 \%$ of participants believed that developers are required to put in a lot of analysis and effort which at times becomes extremely complex and costly. Most businesses restrict their focus to a single platform which reduces the reach of their applications. For cross platform development, there are chances of less documentation, in addition, maintaining and keeping applications updated across multiple platforms with limited resources is quite challenging.

Varying hardware complexities: Mobile phone hardware constantly changes with respect to memory, speed, graphics processing, etc. Furthermore, the processing capability of mobile devices in terms of computational power, processing speed, limited power availability, real time data streams, and memory capacity is much limited as compared to desktop computers. Mobile developer participants have highlighted this as a potential challenge because while developing a mobile app, they have to disable few utilities used by multimedia and graphic mobile apps as they require a large amount of memory and fast processing speed. Hence, mobile developers find it challenging to keep mobile apps capable of error-free operation in both high-end as well as low-end hardware devices.

\section{Challenges related to Software}

Inexperienced resources: When building a mobile app, it is important to follow the style guidelines and behaviour of each mobile device. Less than $10 \%$ of participants showed their concern on lack of knowledgeable and experienced resources to code for variety of mobile devices. In addition, they believed that cultural difference in team may bring conflicts and diversion in team work which may not result in creation of successful mobile app.

Insufficient \& uncertain requirements: Another challenging factor reported is the insufficient and uncertainty in requirements as project can easily get taken off track if business is not clear on the iterative requirements. Approximately $25 \%$ of participant's finds it considerably challenging to assess the effort required at the beginning of the cycle and note they spend a lot of time understanding and analysing customer's requirements increasing their workload with developing, integrating, and testing the apps.

Budget and Schedule: The budget issue is one of the key factors why mobile projects fail, mainly due to slow approval process of funding. It is generally mistaken that mobile development projects are small and hence require low budget. Developing a mobile app is in itself a complex process that involves several steps and stages of development, testing and deployment. This process is not only arduous and tedious, but can also prove to be very expensive for mobile app developers. A mobile developer with advance funds for their project works with a free mind, without having to worry about the expenses they would have to incur on their app. Mobile developers working on tight schedules and highly limited budgets to meet deadline are left with two options, either to deliver a poor quality application to meet the deadline, or miss the deadline.

User Experience (UX): Mobile device is unlike desktop computer as gestures, sensors and location data play a key role in many mobile apps. Over $50 \%$ of the participants indicated that the smaller display, screen layouts and different styles of user interaction have a major impact in designing a mobile app. Some enterprise app developers treat mobile devices as just another screen and extend enterprise applications to mobile devices without realizing that user interactions and behaviours are vastly different. This makes for an unintuitive and cumbersome experience for users resulting in low adoption and usage of the application.

User Interface (UI): The user interfaces for mobile apps may get design ideas from traditional applications. But, it must be redesigned to make most effective use of the screen and the mobile user interface paradigm such as widgets, touch, physical motion and keyboard, including both the user input and the associated motion and location information. Mobile developers find it challenging to make best possible use of limited screen space, and user interface design takes on greater importance than ever.

User input technology: A mobile keypad is more suited for number entry instead of text entry where small buttons and labels limit user efficiency in entering data. Moreover, mobile devices have the capability to receive input from in-built sources, such as GPS and camera besides directly receiving the input from the end user. The input methods offered for mobile devices are intricate and require a certain level of proficiency. Identifying the non-keyboard ways to gather and deliver information to the user is a relatively challenging when building a mobile app.

Form factors: Survey respondents believed small screen size and low screen resolution to be quite challenging while developing a mobile app. A smaller form factor means that fewer amounts of data can be displayed at one time and user requires clarity, simplicity and expects to find the information effortlessly. In addition, low display resolution can degrade the quality of multimedia information displayed on the mobile device screen. As a result, developers are required to put lot of time and effort to design and display the most relevant information required by user at the point in the application.

Data access: Backend integration to enterprise services was reported as one of the major mobile app development concerns by the participants as it requires lot of prior research, planning, and actual development 
work. A mobile device has the capability to access data, either through a web browser or through a native app. If data exists in a backend database, the application should be designed in such a way that it keeps track of lost network connection time so that it can roll back to its last known data and synchronize as soon as connection is resumed back to update the data.

Delivering quality applications: Mobile users expect high quality apps delivered on both design and content front which are quite challenging as many businesses can suffer if customers are influenced negatively by lowquality apps. For this, intensive testing and formal reviews are required to ensure that an app meets all its requirements and offers high quality software before its release to customers.

Complexity of testing: With so many devices and operating system variants, as per $30 \%$ of participants, testing also becomes challenging. Besides the variability in hardware device, there are several factors that need to be addressed for a good user experience, such as wireless switching, VPN drop/restarts, and switching between applications. The test scenarios must not only be implemented on emulators, but also on actual devices to test the success or abrupt crash/failure of mobile apps by identifying any complications due to network connectivity (3G/4G/Wi-Fi/offline) or device hardware (memory/processor).

Analysing the target users: Mobile developers usually hesitate in developing a new application without knowing the target users and their interest upfront. Hence, the failure to identify and analyse potential users and their preferences leads to the development of an app that has something in substance, but nothing in value for the target market with tough competition.

Security and Privacy: The ability to access information via mobile devices can bring immense opportunity, but at the same time information might be misused by unauthorized user. $20 \%$ of participants find mobile device and application security challenging to manage with so many different devices and operating system variants, especially in industries that are heavy regulated such as finance, healthcare, etc. It is the responsibility of mobile developer to preserve data, protect data, and secure the mobile app from end-to-end, including the software, as well as data transmission and servers.

\section{Mobile Application Development - Best Practices}

The paramount results for mobile developers come from applying best practices which involves systematic steps by carefully employing from conceptual to completion stage of mobile application development process. Following practices should be considered for the culmination of the successful development of mobile applications.

Planning: Participants stated that the formulation of the design methodology is a key to the development of mobile applications, mainly in a cross-platform environment where multiple efforts may be in progress simultaneously. Before developing the actual mobile application, mobile companies should have a welldefined reason to create one. In addition, prior research should be done to ensure that it is simply not recreation of an existing app and add extra feature, utility and productivity. For this purpose, a proper plan should be designed with a specified set of actions, thus allowing efficient strategy to attract the potential users.

Requirements: Business plan and logic should be discussed after gathering, analysing and documenting the client's requirement and as per participants, it as the most important phase in the entire mobile software development life cycle. $15 \%$ of participants suggested empowering users by allowing them to choose and control the way their data is collected and used, by maintaining the simplicity and clarity of the application from customer's perspective. The planning strategy should include best user interaction, performance, and limited resource utilization, followed by frequent and rapid iterations of requirements reviews to have respect for context.

Design and architecture: As per one response, "In order to design the best solutions, the team should keep the design simple without consuming too many resources, analyse it and create a simple suitable architecture for the mobile applications". According to $30 \%$ of participants, the best method is to develop a layered application, where the functionality remains consistent across platforms while still meeting the requirements of each platform's look and feel. Modular pieces for each platform should be built where identical business rules and application logic should be retained. As per participant X, "Use wireframes to map out the user experience and allow early discussion of this with the customer. Use frameworks that allow the application to be easily modified and reconfigured whilst in the hands of users. Release early and often."

User Experience (UX): Over 50\% stated that designing a prototype to get an excellent user experience is a top priority and can help make an application more successful by maximizing acceptance and usage. Mobile developers must dedicate time to research on most effective text and graphics that are globally recognizable as well as culturally sensitive. They should use frameworks that allow the application to be easily modified and reconfigured whilst in the hands of users. The application design must present easy access and navigation, making users feel comfortable by providing fast-loading content and intuitive buttons without any tutorial or additional help. It always a good practice to study and analyse the UI design guidelines provided and final user interface and wireframes should be used to map out the user experience to allow early discussion with the customer.

Development skills: The developers should understand every single mobile platform, their ergonomics, and should be aware of the application requirement being targeted. There should be cross-platform development as much as possible by creating reusable codes to speed up 
application development. Developers should participate in product design to decide built in features and determine other features to be added for cross platform development. Few participants recommended an Icebreaker approach and suggested to keep number of features light for first version to develop for a single platform first and later port on the other platforms. In general practice, reviewing user's requirement, designing wireframes, and recoding after user testing is done iteratively where the budget and time frame is fixed for the entire mobile application development process.

Agile practices: $25 \%$ of participants strongly believed that agile approaches (such as, XP, Scrum) should be a practiced for the successful development of mobile application projects but must be tailored to every team.

The most popular Agile approach practiced by participants are test driven development, pair programming, daily scrum meetings, automated testing, continuous integration, iterative and incremental development.

Testing: Application testing should be on real devices, especially with real users in the project for usability, and power consumption to verify they are not battery hogs. Testing should be done frequently, iteratively, early and as often as possible. It should also be tested on the device, outside the building, with multiple browsers. In addition, it should also be tested on multiple devices, on variety of platforms for cross-platform compatibility with different screen sizes and different carrier connections, network to confirm that they accommodate both online and offline usage. $50 \%$ of participants suggested following any agile approach by extensively employing TDD, pair programming, regular code reviews, strong QA, constant measurement and optimization post launch with recommendation to follow UAT release, with bug fixing and production release.

Security: Wireless transmission in mobile devices is vulnerable to attack by unauthorized users as they download and upload data wirelessly, in potentially insecure locations. About 30\% of participants believed that mobile developers should not only integrate the platform's inherent security capabilities but should also consider encryption of sensitive data across open or unsecured networks. As per one participant, "For security measure, always use appropriate and up-to-date security measures to protect user data by using security features such malware apps and Norton antivirus." Mobile developers should register the mobile app to company's name so that any future legal problems may be avoided.

Privacy policy: Mobile platforms may have privacy policy but they are mostly ambiguous. There should be prominent and transparent privacy policy covering data collection, sharing, and use practices. Mobile developers should introduce a policy regarding use of components across platforms, which facilitates maintenance and extensibility across applications on different platforms. Basic steps suggested by participants towards building privacy into mobile apps as follows: practice privacy by design, communicate openly \& effectively, use clear and simple language, make privacy policy easily accessible, use enhanced notice, provide users with choices \& controls, empower users, secure users data and ensure accountability.

Application analytics: The performance of mobile app in the market should be monitored by an Analyst. This will help in knowing additional users requirement that could be further added as a new feature for later updates. Moreover, analytics can also confirm the features that are not used in the way developers expected, those can either be removed or made easier to access in future updates.

Explicit feedback mechanism: Participants are avoiding fire and forget approach and the proper collection of feedback is thus a key component for mobile application to be successful. It is always a good idea to get genuine feedback and review from the users through emails, social networking, application itself, bug reports, etc. Consolidating this response will confirm which features or capabilities, most users are demanding. This will also reduce technical support issues, will result in improvements and will provide insight for future application updates.

\section{DISCUSSION}

The limitations and known challenges and threats to the study are that responses were received only from people willing to be surveyed. There is a risk of getting responses from people with strong feelings about the area as questions capture opinions, not facts. The biases of the communities may be reflected in the results which mean that the survey sample may not represent the real world, and the result may be based on opinion and not fact.

With mobile phones evolving so quickly, long planning and development cycles will result in apps to be out dated and irrelevant though many traditional software engineering techniques may be used in the mobile application domain. In recent time, large and complex software development projects have moved away from process intensive approach towards agile approach. Mobile development projects also require developers to adopt agile methodologies so that they can ship features iteratively, and this can mean reconstructing the software engineering culture within the company. Companies should look beyond the development process to increase their agility so they are in a position to respond faster based on market changes. Excellent collaboration tools have to be used to align teams across the entire software life cycle to overcome time and efficiency constraints.

To provide a solution to the discussed issues and challenges, it has been constantly proposed that agile practices are best suitable and can be tailored for the development of software products for mobile devices. The suitability of Agile methodologies for the development of mobile applications was first discussed in 2003 [9]. Later on, the mapping between agile home ground themes with various development characters were observed and shown in mobile softwares [10]. The mapping demonstrated why agile methods are best 
suitable for implementing development processes in the mobile domain due to small teams, small development cycles, rapidly changing requirements, continuous integration and continuous delivery process.

The agile approach is seen as a natural fit for mobile application development and studies carried out for the application of the agile development approach. This indicates the need for software development processes tailored to suite the mobile application requirements. Prior research works have proved that agile methodologies are one of the best practices to be adhered for mobile software development process [6].

\section{CONCLUSION AND FUTURE WORK}

Our study has given a better, more objective understanding of the real challenges faced by the mobile application developers nowadays, beyond objective stories. The results of this research will contribute towards a greater understanding of mobile software development issues and challenges. After multilateral analysis and investigation of best practices, it is assumed that these can be successfully implemented to mitigate the identified challenges and will enhance the performance of mobile apps when considered during early development process.

The issues, challenges and best practices discussed in Section IV can be considered as possible research topics in mobile application development and can also serve to indicate the breadth of research requirements and prospects in mobile computing. We do not claim that our list of challenges is exhaustive, indeed, we hope that these findings will lead to studies that may result in new challenges being identified, evaluated and addressed that will make this paper a repository of knowledge and a reference for future research on mobile application development. While there are large number of mobile applications, there remains a large number of complex issues where further work is needed. We look at this paper as a working document that can be reviewed and extended with the help of practitioners and researchers. There still remains considerable work to be carried out in this area both in terms of further validation of the underlying best practices, and in more detailed exploration of the proposed mobile application development process.

\section{REFERENCE}

[1] Leigh Williamson, "A mobile application development primer. A guide for enterprise teams working on mobile application projects". IBM Whitepaper. 2012.

[2] Anthony. Wasserman, "Software engineering issues for mobile application development," Proceedings of the FSE/SDP workshop on Future of software engineering research - FoSER", Pages 397-400. 2010.

[3] J. Dehlinger and J. Dixon, "Mobile Application Software Engineering: Challenges and Research Directions". Workshop paper. Oct 2011.

[4] Harleen K. Flora, Xiaofeng Wang, Swati V, Chande, "An Investigation of Characteristics of Mobile Applications: A Survey Study", International Journal of Information
Technology and Computer Science (IJITCS). Communicated. 2014.

[5] Stephen M. Dye, Karen Scarfone, "A standard for developing secure mobile applications," Computer Standards \& Interfaces. 2013.

[6] Harleen K. Flora and Swati V Chande, "A review and analysis on mobile application development processes using Agile," Vol. 3 No. 4. Pages 9-18. 2013.

[7] Ken Schwaber, "Agile Project Management with Scrum" Microsoft Press. 2004.

[8] Harleen K. Flora, Xiaofeng Wang, Swati Chande, "Adopting an Agile Approach for the Development of Mobile Applications," International Journal of Computer Applications, vol. 94, pp. 43-50, 2014.

[9] Pekka Abrahamsson, Warsta, J., Siponen, M., Ronkainen, J., "New directions on Agile methods: A comparative analysis". In proceedings of the international conference on Software Engineering. 2003.

[10] Pekka Abrahamsson, "Mobile software development: the business opportunity of today", in proceedings of the International Conference on Software Development, Pages 20-23. 2005.

\section{Authors' Profiles}

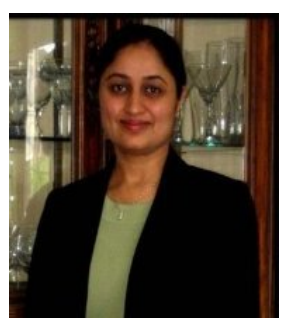

Harleen K. Flora is a research scholar at 'The IIS University', Jaipur, India. She is currently working as an Associate Professor at SBBS Post Graduate College, Jalandhar, India. She is a certified PMP, ITIL Project Manager and holds Master's degree in Computer Management from University of Pune, India. She has over 15 years of extensive experience developing and managing software projects throughout the entire software lifecycle. She worked for Lockheed Martin and served many US government projects as a Consultant. Her research areas include traditional software engineering and Agile software development.

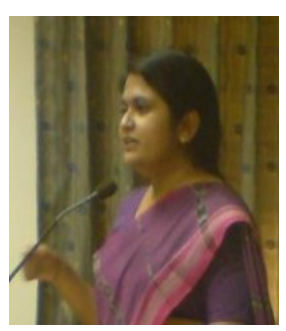

Dr. Swati Chande has over 22 years of experience in teaching, industry, and research. She also has extensive corporate and technical training experience. She completed her M.S. in Software Systems from Birla Institute of Technology and Science, Pilani, Doctorate from Banasthali University, and also has a M.Sc. in Mathematics. Her research interests include database management, genetic algorithms, computational thinking, and software engineering.

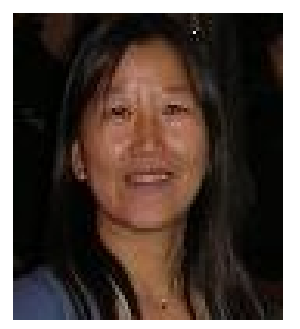

Dr. Xiaofeng Wang is a researcher at the Free University of Bozen-Bolzano, Italy. Her research areas include software development process, methods, agile software development, and complex adaptive systems theory. 